Le Danube traverse le territoire autrichien sur une distance de près de $350 \mathrm{~km}$, ce qui représente environ un septième de la partie navigable. longue de $2380 \mathrm{~km}$, qui s'élend de Ratisbonne jusqu'à la mer Noire (fig. 1). En traversant l'Autriche, le fleuve doit, sur des troncons totalisant environ $150 \mathrm{~km}$, se fraver un passage à travers la montagne (la vallée de Passau: Passau-Aschach; la région du Strudengau: Grein-Ybbs; la Valachie: Melk-Krems; la Porte de Vienne, en amont de Vienne, et la Porte de Pannonie, près de Hainburg) alors que sur près de $200 \mathrm{~km}$, il s'écoule à travers des fonds de vallée plats. Sa pente est de l'ordre de

* Directeur de l'Administration Fédérale des Aménagements des Cours d'Fau, Vienne (Autriche).

\section{LA \\ RÉGULARISATION DU DANUBE
AUTRICHIEN DANUBE
AUTRICHIEN}

\author{
PAR \\ F. TSCHOCHNER *
}

$155 \mathrm{~m}$, son caractère celui d'un fleuve de monlagne a fort charriage. Alors que dans les parties où le fleuve s'écoule à traver's la montagne, il a conservé son lit naturel, le Danube se divise dans les plaines pour y former de nombreux bras, el il a creusé son lit là où il rencontra le moins de résistance. Ceci eut pour conséquence de nombreuses inondations qui, avec les embâcles de glace, causèrent des dommages considérables.

Jusqu'à 1850 environ, le fleuve ne comnut aucune régularisation organisée; on s'était borné à protéger, par des mesures locales, les agglomérations et régions riveraines contre les destructions dues aux crues et aux charriages de glace. Partiellement, cependant, le fleuve fut l'objet de travaux visant l'amélioration du chenal navigable. Ce fut le cas

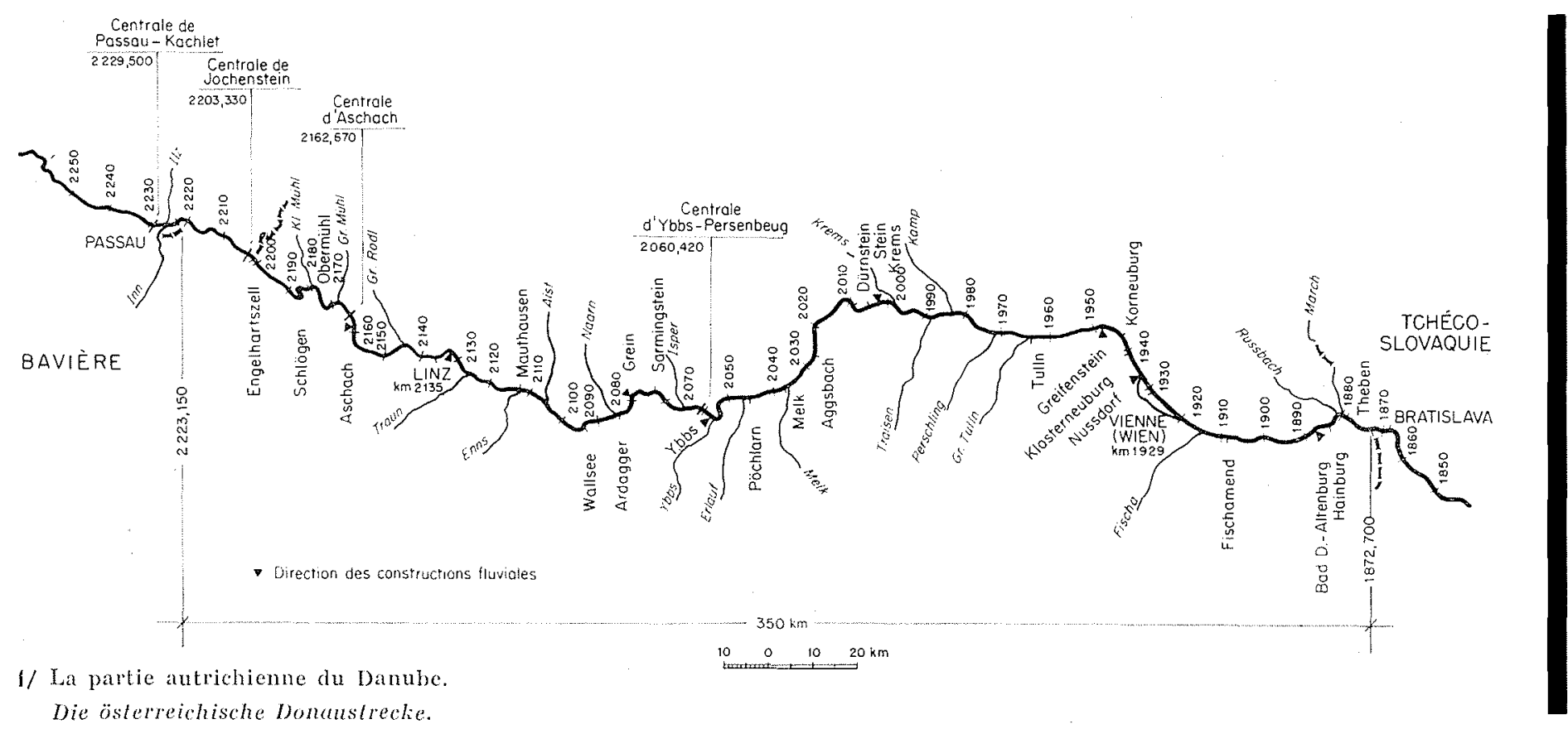

Die östereichische Donalstrecke. 


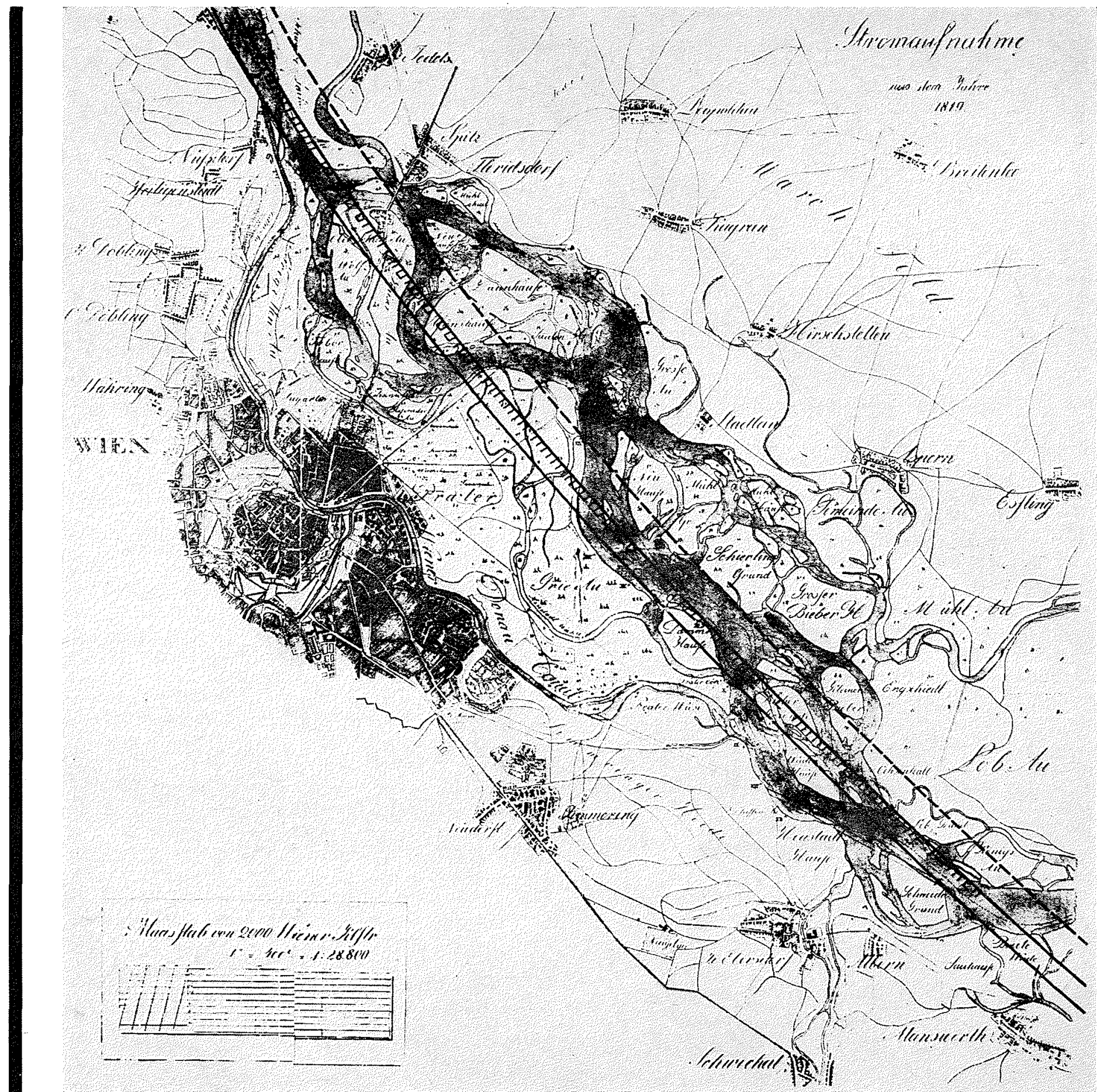

2/ Le Danube dans la région de Vienne.

avec indication de la régularisation des moyennes et basses eaux

et de la digue de protection contre les crues (indiquée en pointillé).

Die Donal im Ranme von Wien, mit eingezeichneter Mittel-

und Niederwasserregulierung und Hochwasserschutzdamm (strichliert).

dès les $X I v^{\prime \prime}$ et $X v^{*}$ siècles dans la région de Vienne, et vers le milien du Xrur" siecle, à l'instigation de l'impératrice Marie-Thérése, dans la zone des rapides près de Grein.

Les débâcles catastrophiques de février 1830 , ainsi que la crue de 1862 conduisirent l'Administration à entreprendre des travaux de régularisation, plus particulierement dans la région de Vienne, et à domner au fleuve un lit uniforme, surtout pour les débits moyens. Les travaux de régularisation dans la région de Vienne, qui conslituèrent laménagement hydraulique le plus imporlant de la vieille Autriche, furent réalisés au cours des années 1869 à 1884 . Plus particulièrement, ils eurent pour but de rapprocher le fleuve de la ville, de créer, pour la navigation, des points de transbordement, et d'écarter Je risque d'inondations dues aux crues et aux amoncellements de glace. Le nouveau lit recut une largeur de $284 \mathrm{~m}$; sur la rive gauche, il est prolongé par une zone d'inondation large de $474 \mathrm{~m}$ et Jimitée par une digue en terre Le troncon a régulariser avait une Jongueur de $26 \mathrm{~km}$, et il nécessila deux percées d'une Iongueur de 6,6 et de $2,5 \mathrm{~km}$, an cours desquelles environ 12 millions de metres cubes de terre durent ère évacués (fiğ. 2).

Par la suite, on entreprit également la régularisation des autres parties du Danube: des bras 
secondaires furent supprimés; on atténua, par-ci, par-là, au moyen de percées et de digues parallèles, des condes brusques; les berges, enfin, furent prolégées par des revètements. En général, ces revètements de berges sont constitués d'enrochements déversés selon une pente de $2 / 3$, et ils dépassent denviron $50 \mathrm{~cm}$ à $1 \mathrm{~m}$ les caux moyennes. Leux largeur de couronnement va de $1,50 \mathrm{~m}$ a $2 \mathrm{~m}$. Entre la cole supérieure du revêtement et le sommet de la berge, la protection est assurée par un perré, d'une épaisseur de $30 \mathrm{~cm}$ environ, établi à la pente de $1 / 2$. Au cours des années, ces revêtements artificiels ont donné lieu à des atterrissements naturels et, de nos jours, la végétation les recouvant donne l'impression - surtout si l'on considère Pensemble que forment le fleuve et les plaines d'inondations boisées - que le fleuve n'a jamais eté touché par la main de l'homme.

La création, vers 1900 , de ce lit des eaux moyennes représenta un progrès considérable dans lo domaine de l'évacuation des eatux des crues et des glaces. Il est certain que, depuis 1893, aucun amoncellement de glace n'a pu ètre constaté dans to lit libre du fleuve. Les poussées de glace qui ont pu ètre observées étaient entièrement dues aux embacles qui se sont produits en aval de la frontière teritoriale.

Cependant, cette régularisation des eaux moyenhes ne pouvait suflire aux exigences toujours plus grandes de la navigation. En hiver, alors que le Ileuve ne présente qu'un faible débit, le lit était trop large pour que la profondeur du chenal navigable fût suffisante. La régularisation de l'étiage fut done entreprise, et elle se poursuit partout où the telle mesure se montre nécessaire.

Cette régularisation de l'éliage est obtenue par un rétrécissement du lit des eaux moyennes au hoyen d'ouvrages longitudinaux et transversaux, de facon qu'aux «eaux basses de régularisation» (RNW) il reste une profondeur d'eau d'au moins $20 \mathrm{dm}$. Pour des niveaux plus faibles, la hauteur d'enfoncement des chalands chargés doit être rétuite dans les mèmes proportions. Le RNW est dćlni comme étant le niveau correspondant à un Acbit qui, au cours des derniers 56 ans, a été atteint ou dépassé en moyenne pendant 340 jours par an. Les ouvrages longiludinaux (digues parallèles) sont des massifs en enrochements, leur largeur de crête est de $2 \mathrm{~m}$ environ, les talus sont aménagés à la pente 2/3. Les ouvrages transversaux (épis) sont tgalement en enrochements, avec une largeur de crète de $2 \mathrm{~m}$ et des talus présentant, côté amont, une pente de $4 / 5$ et, coté aval, une pente de $1 / 3$. Dans les chenaux profonds sont aménagés des sevils de fond dont la crète est au moins à $4 \mathrm{~m}$ an-dessons du RNW.

Dans la région de Vienne, la régularisation de l'étiage fut entreprise au cours des années 18981899. Les installations de transbordement étant loutes situées sur la rive droite, il fut nécessaire, sur une longueur d'environ $20 \mathrm{~km}$, de fixer le chenal navigable à cette berge et de lui assurer une profondeur suffisante. Dans ce but, on construisit des épis qui, implantés sur la rive gauche, s'avancaient vers le milieu du fleuve, ce qui permit de réduire le chenal navigable à une largeur d'environ $180 \mathrm{~m}$. Le succès de cette entreprise se manifesta très rapidement et il s'est confirmé jusqu'à nos jours (fig. 2). Comme exemple des régularisa- lions de l'étiage obtenues au cours des dernières années, nous citerons l'aménagement près de Wilhering, au $\mathrm{km} 2144$ (fig. 3).

Les travaux de régularisation efrectués sur le Danube autrichien, dépendent de l'Administration fédérale de l'Aménagement des Cours d'Eau auprès du Ministère du Commerce et de la Reconstruction; ils sont réalisés par le Service fédéral des Constructions fluviales, organe subordonné à ce Ministère. Pour l'exécution de ces travaux, ce Service a à sa disposition 8 Directions des Constructions lluviales (Aschach, Linz, Grein, Ybbs, Krems, Greifenstein, Vienne, Bad Deutsch-Altenburg) comportant les ateliers, chantiers et carrières nécessaires. De plus, elle dispose d'une Direction des Travaux, équipée de tous les engins nécessaires. Vers la fin de l'année 1961, ce parc comprenait les engins flottants suivants :

-..- Bateaux el embarca-

tions à moteur .....

-- Dragues à godets et dragues à grappin. .

-... Elévateurs flottants.

- Chalands pour enrochements de carrière.

-... Chalands pour cailloux. . . ..........

- Chalands à clapets pour enrochements.

- Chalands à clapets pour cailloux. .....

22 unités, puissance totale $3635 \mathrm{ch}$;

8 unités, capacité totale thérique $815 \mathrm{~m}^{3} / \mathrm{h}$;

2 unités, capacité théorique $550 \mathrm{~m}^{3} / \mathrm{h}$;

35 unités, capacité totale de transport $1900 \mathrm{t}$;

6 unités, capacité totale de transport $695 \mathrm{~m}^{3}$;

2 unités, capacité totale de transport $140 \mathrm{t}$;

7 unités, capacité totale de transport $345 \mathrm{~m}^{3}$.

Parmi les dragues citées, on compte deux gran. des dragues à godets (Fasold et Kaplan) faisant partie des élévateurs flottants (Fafner el Ressel); la capacité théorique de ces dragues est de 250 à $300 \mathrm{~m}^{3} / \mathrm{h}$. Fin tant qu'automoteurs, ces engins sont équipés de 2 moteurs Diesel chacun. Pour chacune de ces dragues, un moteur suffit à produire l'énergie nécessaire; la réparation ou la défaillance d'un moteur n'entraine done aucune interruption dans le travail. En ce qui concerne la drague Fasold, construite il y a environ 35 ans, la profondeur maximale de dragage est de $6 \mathrm{~m}$ au-dessous du niveau d'eau; celle de la drague Kaplan mise en service en 1961 (fig. 4), est de $12 \mathrm{~m}$ au-dessous du niveau de l'eau, ceci compte tenu plus particulie. rement des dragages nécessaires dans les retenues des centrales.

Les élévateurs (fig. 5) sont des engins flottants jumelés; les chalands à cailloux chargés par la drague se glissent entre les deux engins et sont alors déchargés au moyen d'un élévateur a godets; le matériau dragué est amené à la terre par des convoyeurs d'une longueur de 38 ou de $44 \mathrm{~m}$. La hateur maximale de déversement est d'environ $15 \mathrm{~m}$ au-dessus du niveau de l'eau.

La totalité des matériaux dragués est de:

- 2,3 millions de mèlres cubes pour les années 1946 à 1956 , et de

-. 3,7 millions de mètres cubes pour les annes 1957 à 1962 . 


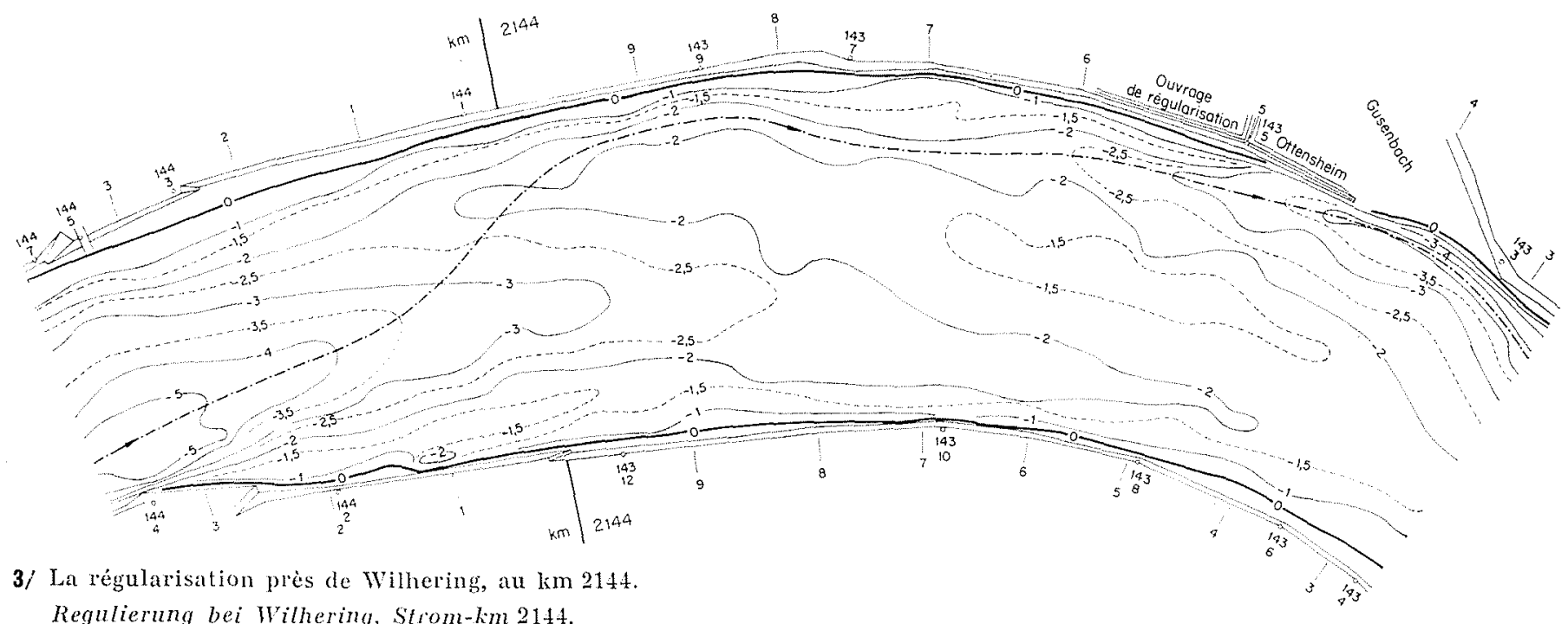

Regulierung bei Wilhering, Stom-km 2144.

En hat : Relevés du fond du fleuve, effectués en 1958 (avant le début de la construction). Oben : Stromgrundatunahme vom Jahe 1958 (vor Baubeginn).

Réalisations de lá construction au cours des années 1958 et 1959 : Dragage d'une cunctte d'une largeur de $80 \mathrm{~m}$, à $2,50 \mathrm{~m}$ au-dessous du RNW; aménagement d'un tere-plein pour le nouveau débarcadère sur la rive droite; construction de trois épis sur la rive droite.

Faudurchführung in den Jahren 1958 and 1959 : Baggerung einer $80 \mathrm{~m}$ breiten Künette, $2,50 \mathrm{~m}$ unter RNW; Anschüttung fïr die neue Schiffsanlegestelle am rechten Ufer; Ban von drei Buhnen am rechlen $\mathrm{Eler}$.

En bas : Relevés du fond du fleuve, au mois d'octobre 1961.

Unten : Stromgrandaluahme bom Olitober 1961.

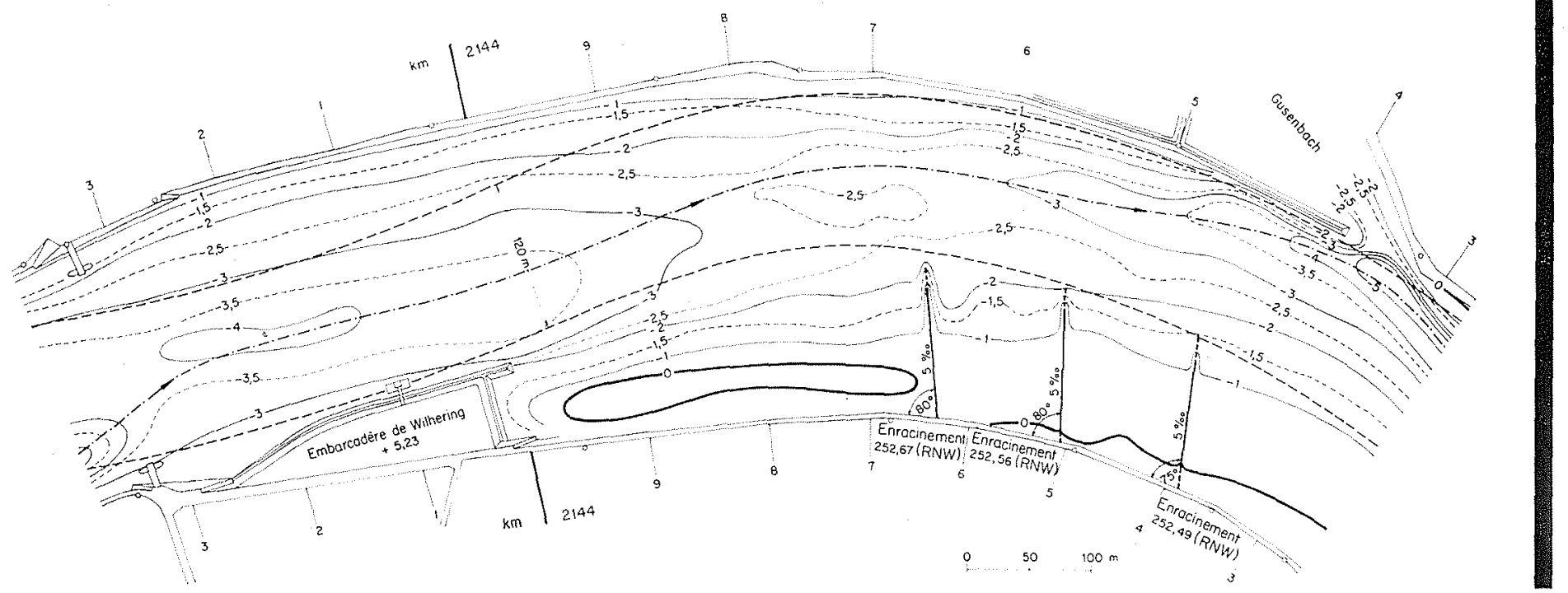

Les quantités d'enrochements utilisées pour l'entretien et la construction d'ourrages de régularisation étaient de :

-..- 776000 tonnes environ pour les années 1946 à 1956 , et de

- 392000 tonnes environ pour les années 1957 à 1962.

En outre, le Service fédéral des Constructions fluviales dispose d'un engin spécial qui, mis au point pour les travaux dans le tronçon dit « Aschacher Kachlet $»$ permet de contrổer si, lors du RNW, chaque point d'un tronçon déterminé du fleuve présente une profondeur d'eau déterminée. Dans l' Aschacher Kachlet», aux km 2159/57, le Danube coule sur un dboulis semblable à la moraine terminale d'un slacier. A cet endroit, le fond est constitué de bloes de roche atteignant jusqu'à $2 \mathrm{~m}^{3}$ et dépassant partiellement le fond du lit. Dans ces conditions, il fut done nécessaure de melle au point un engin pouvant détecter l'emplacement des obstacles et les supprimer immédiatement. Cet appareil dit «engin de sondage» (fig. 6) est constitué de deux coques de bateaux reliées entre elles par des cadres; entre ces deux coques se trouve une benne preneuse destinée à remonter les blocs de roche. Aux bords extérieurs de ces coques de bateaux sont aménagés des cadres de sondage pouvant être réglés à la profondeur désirée, qui, lors des déplacements transversaux de l'engin, indiquent par déviation chaque obstacle. Dans l' «Aschacher Kachlet», environ 15000 blocs de roche ont pu être remontés de cette manière, ce qui a permis d'améliorer considérablement la profondeur du chenal navigable. Aujourd'hui, l'Administration fédérale de l'Aménagement des Cour's d'Fau dispose de quatre engins de ce genre, grâce auxquels on put, par la suppression des blocs de roche, améliorer considerablement les conditions du chenal navigable sur toute la partie autrichienne du Danube.

Les méthodes de régularisation, dont il fut question jusqu'ici, avaient pour but, dans ce fleuve dont 
Peconlement naturel n'est perturbé par aucun ouvage, d'assurer une profondeur suffisante du chenal navigable, el de garantir l'évacuation aussi pen dangereuse que possible des caux des crues et des glaces.

Depuis l'année 1952, des centrales hydroélectriques sont aménagées dans la partic autrichienne du Danube. Pour la centrale de Jochenstein, dont la zone de retenue est immédiatement à l'aval de l'étiage de retenue de Passau-Kachlet qui existe depuis 1927 , le plein de la retenue fut atteint en 1955 ; pour la centrale d'Ybbs-Persenbeug, il fut atteint en 1958. La centrale d'Aschach est actuellement en construction.

La coustruction des centrales marque le début d'une nouvelle époque pour le fleuve el ses environs, mais elle pose avant loul de nouveaux problemes à l'ingénieur hydraulicien. L'Administration fédérale de l'Aménagement des Cours d'Eau ctant toujours responsable du maintien satisfaisant de la navigation et de l'évacuation aussi peu dan gereuse que possible des eaux des crues et des glaces, des voies et moyens doivent ètre trouvés afin de continuer à répondre à ces exigences.

Le régime des solides et des matériaux en suspension se trouve sensiblement afrecte par les onvrages aménagés. Du fait de la retenue et de son cflet sur la vitesse d'écoulement qui, depuis l'enracinement de la retenue jusqu'à l'cuvage principal, diminue progressivement, le fleuve n'est plus à même d'évacuer les solides et les matériaux en suspension, ce qui, inévitablement, conduit à des dépôts dans les zones de retenue.

Des mesures du débit des solides et des matériaux en suspension ont ćté effectuées afin d'obtenir une idee des quantités que transporte le fleuve à l'état non perturbé.

Au cours des années 1956 et 1957 , Ie Service fédéral des Constructions fluviales effectua des mesnres du débil solide dans un profil de gué choisi, situé près de Bad Deutsch-Altenburg, mesures directement motivees par le projet de construction d'une centrale danubienne dans la région de Bratislava. Dans chaque cas, les mesures furent effectuées à l'aide d'une nasse d'Ehrenherger en 10 verticales réparlies sur toute la largeur du fleuve. La quantite des matériaux ainsi prélevés fut déterminée par peséc à l'état sec. Les principales connaissances obtenues par ces mesures du débit solide se résument comme suil : le poids spécifique est de $1,8 \mathrm{t} / \mathrm{m}^{3}$; il y a charriage même pour de fables débits - pour RNW, un charriage a pu ètre constaté sur près de la moilié de la largeur totale du fleuve; le diamètre déterminant des grains, selon Mayer-Peter, est de $13 \mathrm{~mm} ; 76 \%$ ont un diamètre de $15 \mathrm{~mm}$ ou inférieur; pour seulement $0,2 \%$, il est supérieur à $70 \mathrm{~mm}$; une grosseur inférieure à $1 \mathrm{~mm}$ n’a pu ètro constatée. D’apris les résultats de ces mesures, le débit solide pour une année moyenne peut être évalué à $600000 \mathrm{~m}^{3}$.

Dans une large mesure, le rógime des matériaux en suspension est fonction du régime du fleuve. C'est ainsi que l'on a pu constater que jusqu'aux eaux moyennes, le régime des matériaux en suspension augmente selon la puissance 1,5 environ du régime du fleuve; au-dessus des eaux moyennes, au contraire, il augmente selon la puissance 3 à 4 . Compte tenu de cette comnaissance, il suffit done, pour des régimes allant jusqu'à $1000 \mathrm{~m}^{3} / \mathrm{s}$, de ne faire des mesures que trois fois par semaine; pour

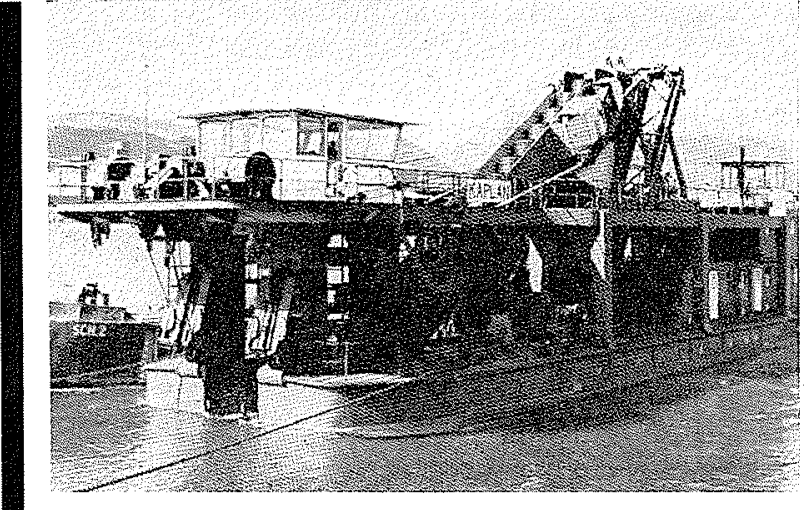

4/ Drague à godets « Kaplan $\%$

Eimerkettenschwimmbagger «Kaplan».

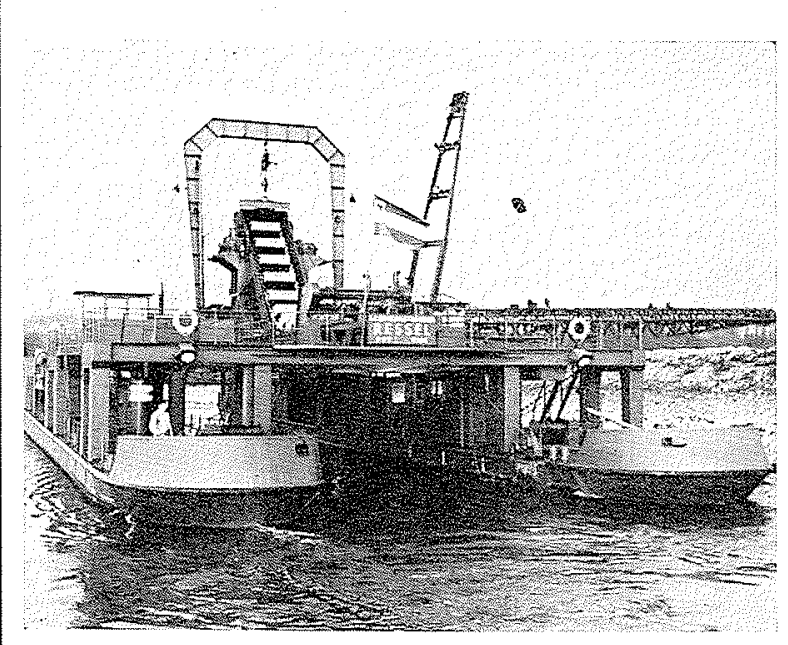

5/ Elévateur flottant «Ressel».

Schwimmender Elevator «Ressel».

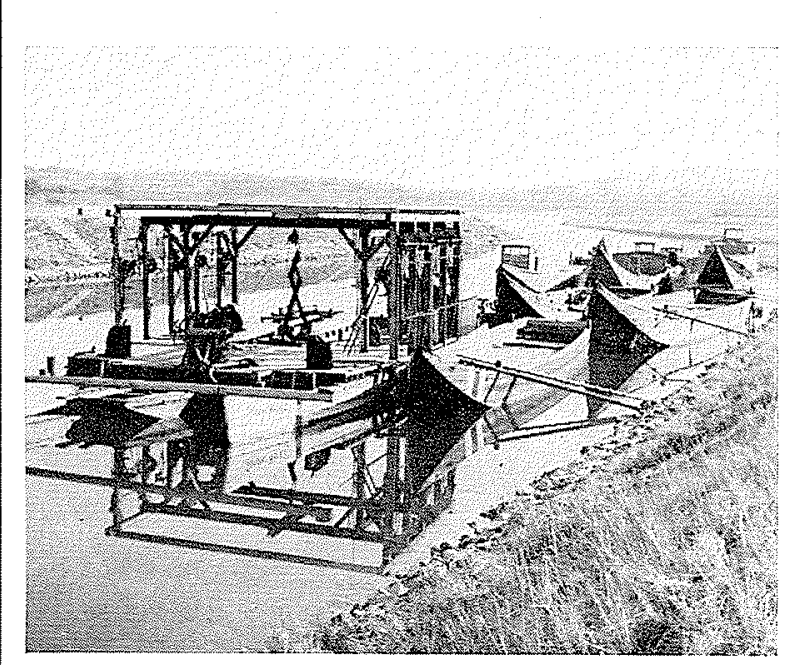

6/ Installation de sondage.

Sondierwerk.

des régimes se situant entre 1000 ot $2000 \mathrm{~m} / \mathrm{s}$, en revanche, il est nécessaire d'effectuer des mesures tous les jours, el pour des régimes dépassant $2000 \mathrm{~m}^{3} / \mathrm{s}$, au moins deux fois par jour.

Les valeurs annuelles du débit des matériaux en suspension sont très dispersées; elles se situent entre 3 el 5 millions de tonnes. Les valeurs les plus importantes furent atleintes au cours des années 1954 et 1959 : la crue catastrophique de 1954 était similaire à celle de 1899 ; en 1959 , aucune hauteur d'eau extrême n'a été constatée, mais l'on a pu observer plusieurs petites ondes de crue. La 


\section{F. TSCHOCHNER}

moyenne du débit des matériaux en suspension peut être estimée ì 4 millions de tonnes $\left(1,35 \mathrm{l} / \mathrm{m}^{3}\right)$.

1l est donc indiscutable que les quantites de matériaux solides et en suspension transportés par le fleuve en amont des retenues des barrages, s'y déposent entierement ou en partie, alors qu'en aval des barrages, le lit du fleuve a tendance à s'approfondir, les matériaux retenus lui faisant défaut.

Il faut donc débarrasser les retenues des matériaux solides et en suspension qui s'y accumulent, dans la mesure où ils gênent la navigation ou l'évacuation des crues. Des dépòts suffisamment grands doivent être disponibles pour pouvoir recevoir les matériaux dragués ne pouvant être utilisés dans l'industrie du bâtiment. Pour l'emploi de ces matériaux dans cette industrie, le facteur déterminant est avant tout la distance séparant le centre d'utilisation du lieu de dragage. Dans le cas où la distance est telle qu'elle entraîne des frais de transport trop élevés, les matériaux dragués doivent ètre déposés de part et d'autre du fleuve, dans d'anciens bras ne jouant plas un ròle important dans l'évacuation des crues; mais il faut alors tenir compte des exigences de la protection des sites.

Pour compenser les matériaux non disponibles en aval, le fleuve en arrache à son lit, ee qui, au cours des temps, conduit à un approfondissement naturel. Labaissement du niveau aval entraine alors une augmentation de la production des centrales. Cet approfondissement à l'aval peut ètre obtenu artificiellement et plus rapidement par des dragages, comme ce fut le cas pour Jochenstein, où le niveau aval fut artificiellement abaissé de 80 à $90 \mathrm{~cm}$ au moyen de dragages.

Mais il est également possible que, en aval, les responsables du barrage soient obligés de prendre des mesures analogues à celles prises pour la centrale d'Ybbs-Persenbeug. Ce barrage existe depuis quatre ans, le plein de la retenue de cette centrale ayant été atteint en novembre 1958. Au cours des annees 1960 et 1961 , à l'enracinement de la retenue, près de Wallsee, environ $750000 \mathrm{~m}^{3}$ ont été extraits par dragages. Le matériau manquant en aval n'étant compensé par aucun apport, un approfondissement naturel s'est créé en cet endroit.

Cependant, près de Sarling, $4 \mathrm{~km}$ en aval de la centrale, un seuil granulitique empêche en cet endroit un approfondissement naturel, sans arrêter pour autant le transport des matériaux. Il s'ensuit que le seuil dépasse de plus en plus le fond de la rivière de sorte qu'à l'étiage, la navigation ne dispose plus d'une profondeur suffisante; de plus, la pente du niveau d'eau augmente. Dans ce cas, il n'est donc pas possible d'attendre que l'approfondissement se fasse naturellement sous l'action du fleuve, et il faut supprimer artificiellement la barre rocheuse. Il est évident qu'au cours des travaux - - ils débutèrent en automne 1962 - la destruction de la barre rocheuse doit se faire immé- dialement, de telle sorte que plus rien ne puisse s'opposer à un approfondissement naturel jusqu'à l'obtention de l'état d'équilibre.

Afin d'éviter le plus possible les amoncellements de glace qui, dans les zones des retenues, se trouvent extrêmement favorisés en raison de la faible vitesse d'écoulenent, il faut, dès l'élablissement du tracé des nouvelles berges, veiller à ce que ce tracé soit aussi net que possible. Comme il est prescrit dans le règlement, chaque étage de retenue doit disposer d'un nombre suffisant de brise-glace pour empecher - ou supprimer - les embâcles. En ce qui concerne l'évacuation des glaces par les pertuis, lon a constaté qu'il est plus efficace de libérer rapidement et pour une durée limitée -- environ une heure - - un ou deux pertuis plutòt que de n'abaisser que peu toutes les vannes, car l'on obtient ainsi rapidement une chute plus forte du niveau de l'eau en amont du barrage. Bien entendu, les efforts auxquels sont soumis les ouvrages s'en trouvent nettement accrus; il est done nécessaire, des la construction du barrage, de donner une résistance suffisante à ces éléments.

Lorsque le débit augmente, les pertuis sont libérés progressivement de telle façon que, lors des crues, toute la section d'écoulement soit disponible. En cas de besoin, les eaux des crues peuvent également ètre évacuées par les écluses.

Comme il ressort de l'exposé fait ci-dessus, ce sont avant tout le transport solide et le transport en suspension qui se trouvent considérablement affectés par l'aménagement de centrales fluviales, et les mesures de régularisation prévues dans les parties soumises à l'influence des barrages doivent donc avant tout tenir comple de ces problèmes. Afin de ramener ces mesures à un volume économiquement raisonnable, il semble judicieux de construire des chaines ininterompues de centrales plutôt que d'aménager des centrales individuelles. Dans une telle chaîne de centrales, Jes dragages des dépôts de solides seront surtout nécessaires près de l'enracinement de la retenue du premier barrage en amont, tandis que les mesures contre des approfondissements ne devront être prises qu'en aval du dernier étage de retenue. Il sera également plus facile de rester maitre du régime des glaces, le risque d'un embâcle n'étant pratiquement à craindre qu'à l'enracinement de la retenue du barrage situé le plus en amont. Enfin, une chaine de centrales présente des avantages considérables pour la navigation, la disposition des trains de bateaux - en ce qui concerne leur composition et la puissance des machines - pouvant rester la mème sur d'assez longues distances.

Au cours des années à venir, l'ingénieur aura pour tâche d'élaborer des méthodes, tant du point de vue des constructions fluviales que de l'exploitation, qui permettront, de manière économique, de ramener le fleuve à un état d'équilibre, mềme dans les zones affectées par les centrales.

\section{Zusammenfassung Die Regulierung der Donau in Oesterreich Von Wikl. Hofrat. Dipl. Ing. Franz Tschochner: *}

Die Donau durchfließt österreichisches Staatsgebiet in einer Länge von rund $350 \mathrm{~km}$, das ist rund ein Siebentel der $2380 \mathrm{~km}$ langen schiffbaren Strecke zwischen Regensburg und dem Schwarzen Meer (Abb. 1). In der österreichischen Donaustrecke entfallen rund $150 \mathrm{~km}$ auf Strecken, in denen der Strom Gebirge durchbricht, rund $200 \mathrm{~km}$ liegen in Talniederungen, das Gefälle beträgt rund $155 \mathrm{~m}$, der Charakter ist der eines Gebirgsstromes mit lebhafter Geschiebeführung:

\footnotetext{
* Leiter des Bundesstrombauamtes, Wien.
} 
Bis zur Vitte des vorigen Jahrhunderts kann von einer planvollen Regulierungstätigkeil nicht gesprochen werden, man sicherte lediglich durch örtiche Jaknahmen Siedlungen und Vfergelände gegen Zerstörungen durch Hochfluten oder Eisgang. Vereinzelt wurden auch Arbeiten zur Verbesserung des Fahrwassers für die Schiffahrt durchgeführt.

Die Eisgangkatastrophe vom Februar 1830 und das Hochwasser vom Jahre 1862 veranlaßten die öffentliche Verwaltung, Stromregulierungsarbeiten, insbesondere bei Wien, in Angriff zu nehmen und der Donau vor allem für mittlere Wasserführungen ein einheitliches Bett zuzuweisen.

Anschließ̧end ging man daran, auch die übrige österreichische Donaustrecke zu regulieren : Nebenarme wurden abgebatu, da und dort scharfe Krümmungen durch Durchstiche oder leitwerke beseitigt, die Ufer wurden durch Deckwerke befestigt. Diese Uferdeckwerke bestehen in der Regel aus einer 2 : 3 geneigten Bruchsteinschüttung bis etwa $1 / 2$ bis $1 \mathrm{~m}$ über Mittelwasser mit 1.5 bis $2 \mathrm{~m}$ Kronenbreite, anschließend daran ist bis zum Ufergrat cine etwa $30 \mathrm{~cm}$ starke Pflasterung $1: 2$ hergestellt. Diese künstlich hergestellten Uferdeckwerke haben sich im Laufe der Zeit auf natürliche Weise verlandet und bewachsen, so daß heute der Strom, besonders in Verbindung mit den dahinter liegenden Auwäldern, einen durchaus naturbelassenen Eindruck macht.

Mit der Schaffung dieses Mittelwasserbettes war etwa um die vergangene Jahrhundertwende ein gewaltiger Fortschritt hinsichtlich Hochwasser- und Eisabfuhr erreicht. In der österreichisehen Donaustrecke hat sich jedenfalls seit $1893 \mathrm{im}$ freien Strom keine Eisversetzung mehr gebildet. Aufgetretene Eisstöße waren ausnahmslos auf Eisstauungen zurückzuführen, die stromabwärts der Landesgrenze eingetreten sind.

Den ständig steigenden Anforderungen der Schiffahrt genügte diese Mittelwasserregulierung jedoch nicht. Im Winter bei geringer Wasserführung. war das Bett zu breit für eine ausreichende Fahrwassertiefe. Es wurde daher, und wird laufend, wo notwendig, eine Niederwasserregulierung eingebaut. Diese Niederwasserregulierung besteht darin, daß das Mittelwasserbett durch Quer- oder Längsbauten weiter eingeengt wird, sodaß bei « Regulierungsniederwasser $\gg$ (RNW) noch mindestens $20 \mathrm{dm}$ Wassertiefe vorhanden sind. Das RNW ist definiert als jener Wasserstand, der einer Wasserführung entspricht, die in den letzten 56 Jahren im Mittel an 340 Tagen im Jahr erreicht oder überschritten wurde.

Die Niederwasserregulierung im Raum ron Wien wurde in den Jahren 2898-1899 durchgeführt. Da die Umschlagsanlagen ausnahmslos an rechten Stromufer lagen war es notwendig, die Schifahrtsrinne auf eine Länge von rund $20 \mathrm{~km}$ mit ausreichender Fahrwassertiefe an dieses Ufer zu fixieren : es wurden vom linken Ufer gegen die Strommitte Buhnen hergestellt und die Schiffahrtsrinne auf etwa $180 \mathrm{~m}$ eingeengt. Der Erfolg stellte sich rasch ein und ist bis heute erhalten geblieben (Abb.2). Als Beispiel einer in den letzten Jahren ausgeführten Niederwasserregulierung wird die Arbeit bei Wilhering, Strom-km 2144, angeführt (Abb. 3).

Die Regulierungsarbeiten in der österreichischen Donaustrecke unterstehen der Bundeswasserbauverwaltung im Bundesministerium für Handel und Wiederaufbau und verden vom Bundesstrombauamt, als nachgeordneter Dienststelle dieses Bundesministeriums durchgeführt. Dieses Ant verfügt über einen umfangreichen schwimmenden Gerätepark: Schiffe, Motorboote, Bagger, Elevatoren, Transportkähme und Klappschuten für Steine und Schotter; zwei große schwimmende Baggergarnituren bestehend aus einem Eimerkettenbagger und einem Elevator mit Förderband besitzen eine Leistungsfähigkeit von je 250 bezw. $300 \mathrm{~m}^{3} / \mathrm{h}$ (Abb. 4 und 5).

Die gesamten Baggerleistungen betrugen in den Jahren

1946 bis $1956 \mathrm{rd}$. $2.3 \mathrm{Mio} \mathrm{m}^{3}$

1957 bis 1962 rd. 3.7 Ulio $\mathrm{m}^{3}$,

an Bruchstein für Erhaltung und Neubau von Regulierungswerken wurden verbaut:

in den Jahren $1946-1956 \ldots \ldots \ldots \ldots \ldots$. r . $776000 \mathrm{t}$

in den Jahren $1957-1962 \ldots \ldots \ldots \ldots \ldots \ldots$ rd. 392000 t.

Zum punktweisen Absondieren des Strombettes verfügt das Bundesstrombatamt schließlich über ein Spezialgerät. Dieses Gerät, genannt Sondierwerk (Abb. 6), besteht aus 2, durch Rahmen verbundene Schiffskörper, zwischen denen sich eine Greiferzange zum Heben der Hindernisse befindet. An den äußeren Seiten der Schiffskörper sind Sondierrahmen angebracht, die auf die gewünschte Wassertiefe eingestellt werden können und bei Querfahrten des Gerätes jedes Hindernis durch Ausschlagen anzeigen.

Bisher wturden die Regulierungsmethoden behandelt, die zum Ziele hatten, in dem durch keinerlei Einbauten in seinem natürlichen Abflußvermögen gestörten Strom einerseits die für die Schiffahrt notwendigen Fahrwassertiefen sicherzustellen, andererseits die möglichst unschädliche Abfuhr von Hochwasser und Eis zu gewälrleisten.

Seit dem Jahre 1952 werden in der österreichischen Donaustrecke Wasserkraftwerke errichtet. Damit beginnt für den Strom und seine Umgebung ein neuer Zeitabschnitt, insbesondere entstehen für den Strombauingenieur neue Probleme. Da die Bundeswasserbauverwaltung weiterhin für die klaglose Aufrechterhaltung der Schiffahrt und die möglichst unschädliche Abfuhr von Hochwasser und Fis verantwortlich ist, mïssen Mittel und Wege gefunden werden, um diese Forderungen weiterhin zu erfüllen.

Durch die vorgenommenen Einbauten wird das Schwer-lund Schwebstoffregime empfindlich beeinflubt.

Um ein Bild über die Mengen zu erhalten, die der Strom im ungestörten Zustand transportiert, wurden Geschiche- und Schwebstoffmessungen durchgeführt. Nach diesen Messungen beträgt die Geschiebefracht des mittleren AbfluBjahres rund $600000 \mathrm{~m}^{*}$, dig Schwebstofführung etwa 4 Mio t. Geschiebeablagerungen an der Stauwurzel müssen, sofern sie entweder hinderlich für die Schiffahrt sind oder eine nicht tragbare Hebung des Hochwasserspiegels herbeiführen, entfernt werden. An der Stauwurzel des Kraftwerkes Ybbs-Persenbeug wurden in den Jahren 1960 und 1961 rund $750000 \mathrm{~m}^{3}$ Schotter gebaggert. Die im Unterwasser eines Kraftwerkes durch den Gesehiebemangel entsehende Eintiefuns kann entweder künstlich herbeigeführt werden, wie dies beim kraftwerk Jochenstein geschehen ist, oder sie wird dem Flub überlassen. Etwa im Unterwasser ausgespülte Felssehwelen, die dann ein Hindernis für die Schiffahrt bilden, müssen entfernt werden, wie dies beim Kraftwerk Ybbs-Persenbeug der Fall ist. Zur möglichsten Verhinderung von Eisversetzungen muß schon bei der Ausgestaltung der neuen Ufer auf eine möglichst flüssige Linienführung Bedacht genommen werden. Außerdem wird bei jeder Kraftstufe die Bereithaltung von Eisbrechern \%wingend vorgeschrieben. Zur Hochwasserabfuhr stehen neben den geöfneten Wehrsehützen für den Notfall auch die Schiffsschleusen zur Verfügung.

Da durch den Bau von FluBkmaftwerken vor allen das Schwer-, Schwebstofl- und Eisregime empfindlich beeinflußt wird, müssen die Regulierungsmaßnahmen in den durch Kraftwerke beeinflußten Strecken in erster Linie auf diese probleme abgestellt werden. Um die erforderlichen Maßnahmen auf ein wirtschaftlich erträgliches Ausmaß zu reduzicren erscheint es zweckmäßig, nicht Einzelkraftwerke sondern geschlossene Kraftwerksketten zu errichten.

Es wird Aurgabe der nächsten Jahre sein, flußbau- und betriebstechnische Methoden auszuarbeiten, um den Strom auch in den von Kraftwerken beeinfluBten Strecken auf die wirtschaftlichste Weise wieder in den Gleichgewichtszustand zurückzuführen. 


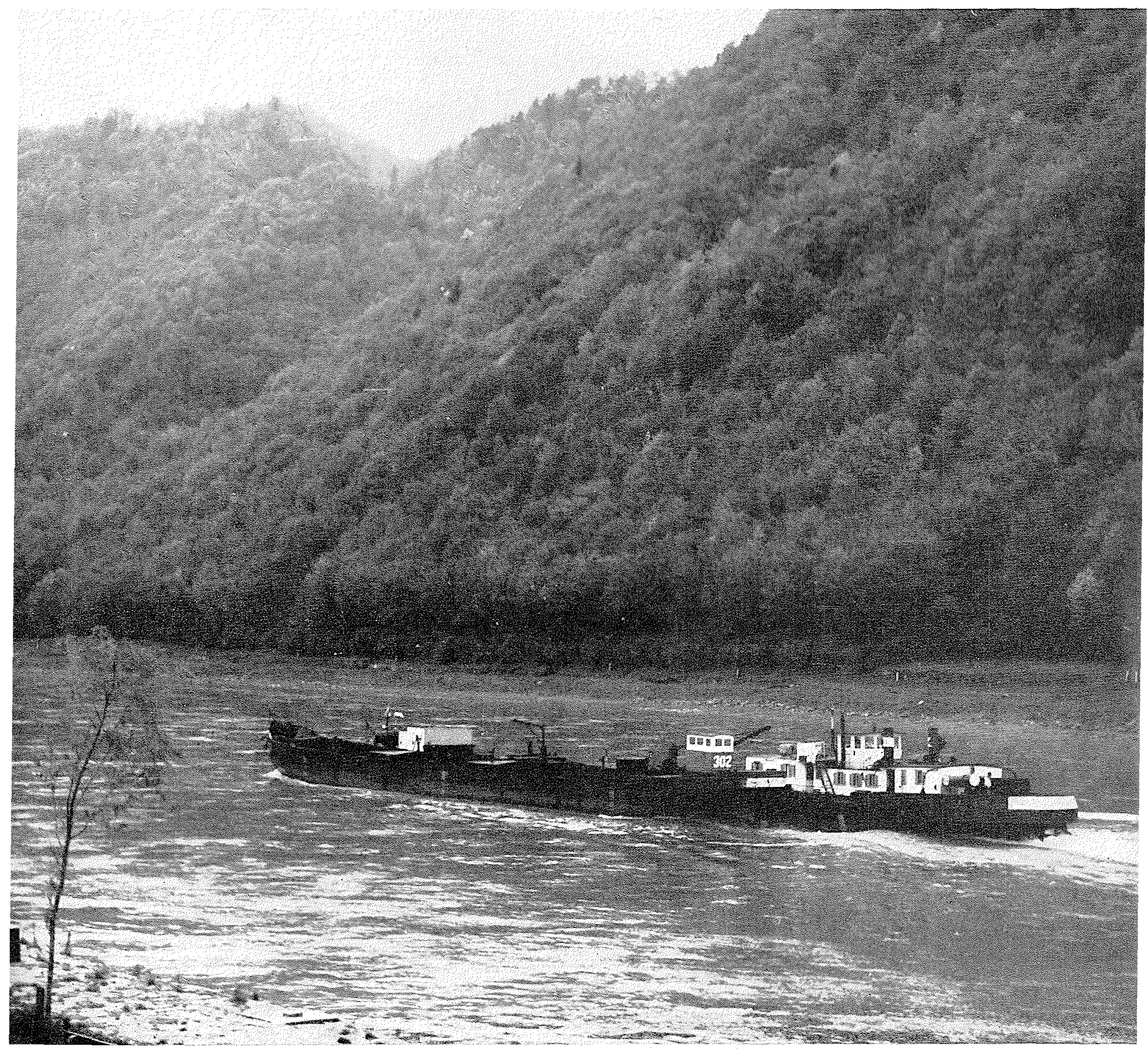

